\title{
Emociones y difusión de noticias sobre el cambio climático en redes sociales. Influencia de hábitos, actitudes previas y usos y gratificaciones en universitarios
}

\author{
Emotions and news on social media about climate change sharing. \\ Moderating role of habits, previous attitudes and uses and \\ gratifications among University students
}

Francisco Segado-Boj. Universidad Internacional de La Rioja. España.

francisco.segado@unir.net

$[\mathrm{CV}]$ (1) $\mathrm{G}$

Jesús Díaz-Campo. Universidad Internacional de La Rioja. España.

jesus.diaz@unir.net

[CV] 7

Nuria Navarro-Sierra. Universidad Rey Juan Carlos. España.

nuria.navarro.sierra@urjc.es

$[\underline{\mathrm{CV}}]$ (1) $\mathrm{G}$

Investigación financiada. Este artículo es producto del proyecto de Investigación titulado "Consumo de noticias en medios sociales. Análisis de factores en la selección y difusión de contenidos mediáticos", referencia CSO2017-86312-R financiado por el Ministerio de Economía, Industria y Competitividad (MINECO), la Agencia Estatal de Investigación (AEI) y el Fondo Europeo de Desarrollo Regional (FEDER), dentro de la convocatoria 2017 de ayudas a proyectos de $I+D+I$ correspondientes al Programa Estatal de Investigación, Desarrollo e Innovación Orientada a los Retos de la Sociedad en el marco del Plan Estatal de Investigación Científica y Técnica y de Innovación 2013-2016. Fechas: Inicio de la investigación: 15 de marzo de 2017. Término de la investigación: 20 de septiembre de 2018.

Cómo citar este artículo / Referencia normalizada

Segado-Boj, F., Díaz-Campo, J. y Navarro-Sierra, N. (2020). Emociones y difusión de noticias sobre el cambio climático en redes sociales. Influencia de hábitos, actitudes previas y usos y gratificaciones en universitarios. Revista Latina de Comunicación Social, 75, 245-269.

https://www.doi.org/10.4185/RLCS-2020-1425

\section{RESUMEN}

Introducción: Esta investigación analiza la influencia que ejercen las emociones a la hora de decidir si se comparten las noticias sobre el cambio climático en redes sociales. Se estudia la función moderadora de los hábitos mediáticos, las actitudes previas y los usos y gratificaciones en esa decisión. Metodología: Se remitió un cuestionario online a una muestra de estudiantes universitarios de distintas titulaciones y centros de la Comunidad de Madrid. Los datos obtenidos fueron tratados estadísticamente según los modelos de regresión lineal simple y múltiple, de regresión logística 
simple y múltiple, y de regresión ordinal simple y múltiple. Resultados: El miedo y la rabia influyen en que una noticia relativa al cambio climático se comparta o no. La búsqueda de información, el consumo de noticias y el grado de preocupación previa son factores que moderan esa influencia.

PALABRAS CLAVE: cambio climático; redes sociales; emociones; teoría de los usos y gratificaciones; hábitos de consumo informativo.

\begin{abstract}
Introduction: This article analyzes the influence of emotions on users' intention to share news about climate change on social media. Media consumption habits, previous attitudes towards the issue and social media uses and gratifications sought are considered as moderating roles. Methodology: An online, self-administered, questionnaire was submitted to a sample of undergraduate students from different courses and centers placed at Madrid region. Data were statistically tested following simple and multiple linear regression, simple and multiple logistic regression and simple and multiple ordinal regression models. Results and conclusions: It is concluded that fear and anger are the most influential emotions on users' intention to share a piece of news on social media. Information seeking, news internalizing and previous attitudes are identified as moderating factors.
\end{abstract}

KEYWORDS: climate change; social media; emotions; uses and gratifications theory; news consumption habits.

\title{
CONTENIDO
}

1. Introducción, 1.1. Emociones y difusión de noticias, 1.2. Redes sociales y cambio climático, 1.3. Emociones y cambio climático, 1.4. Usos y Gratificaciones, 1.5. Justificación y objetivos, 2. Metodología, 2.1. Tratamiento estadístico, 3. Resultados, 3.1. Influencia de las emociones en la intención de compartir la noticia, 3.2. Papel moderador de los usos y gratificaciones perseguidos, 3.3. Papel moderador de la internalización y externalización de noticias, 3.4. Papel moderador de interés, conocimiento y actitudes previos, 4. Discusión y conclusiones, 4.1. Limitaciones y prospectiva, 5. Referencias bibliográficas, 5.1. Artículos relacionados.

Traducción del resumen Francisco Segado-Boj (Doctor en Periodismo).

\section{Introducción}

El ecosistema mediático contemporáneo se encuentra en profunda transformación (Velásquez et al., 2018). Uno de los cambios más profundos en este sentido es la emergencia de las redes sociales como vehículos para la difusión y el consumo de noticias y otros contenidos mediáticos (Bright, 2016).

Plataformas como Twitter o Facebook se han convertido en un nuevo escaparate y vehículo de distribución de noticias y, en consecuencia, han cambiado el modo en que los ciudadanos acceden a la información y entran en contacto con asuntos de interés cívico y político (Kalsnes \& Larsson, 2018). En el caso concreto de España el $48 \%$ de los internautas utiliza Facebook para leer, encontrar, compartir o comentar noticias (Amoedo, Vara-Miguel, \& Negredo, 2018).

Los usuarios ya no son el punto final de una línea de transmisión, sino nodos dentro de una red, de modo que su función ya no se limita a recibir información de modo pasivo, sino que también participan de manera activa en la difusión y distribución del contenido mediático (Klinger; Svensson, 2016). Este nuevo papel de los usuarios ha sido etiquetado como gatekeeping secundario (Singer, 
2014), contenido distribuido por el usuario (Villi \& Matikainen, 2015) o re-diseminación (Guallar, Suau, Ruiz-Caballero, Sáez, \& Masip, 2016).

Este panorama ha llevado a las compañías mediáticas a diseñar y aplicar estrategias para maximizar la difusión de su contenido en redes sociales. De modo específico, las empresas informativas han utilizado los medios sociales para ampliar el alcance de sus contenidos (Villi \& Noguera-Vivo, 2017) y, de manera paralela, generar una mayor implicación de su público (Martín-Quevedo, FernándezGómez \& Segado-Boj, 2019). Esta tendencia ha llevado a que los medios desarrollen y apliquen nuevas lógicas informativas y valores noticiosos, diferentes a los de los medios tradicionales (Larsson, 2018; Lischka, 2018).

\subsection{Emociones y difusión de noticias}

Dentro de estos nuevos atributos noticiosos enfatizados destaca de manera prominente el aspecto emocional de la noticia, puesto que el contenido que genera una reacción emocional más intensa es más dado a ser compartido por los usuarios. (Kilgo, Lough, \& Riedl, 2017). La reacción emocional de los usuarios es junto a otros aspectos, como la personalidad o los usos y gratificaciones, uno de los factores que explica la decisión de los usuarios de compartir contenido en redes sociales (Dafonte-Gómez, 2018).

Según la Teoría de la Difusión Emocional (o Emotional Broadcaster Theory) los individuos tienen la necesidad innata de compartir experiencias. El compartir noticias emotivas a través de las redes sociales es uno de los modos mediante los cuales se puede satisfacer esa necesidad (Harber \& Cohen, 2005).

En este sentido, el público comparte de manera preferente en Internet aquellos artículos que provocan emociones más intensas (Berger \& Milkman, 2012; Weeks \& Holbert, 2013). Consecuentemente, aquellas publicaciones que expresan algún tipo de emoción se comparten con más frecuencia que el contenido de tono neutral (Keib, Himelboim, \& Han, 2018; Stieglitz \& DangXuan, 2013).

No obstante, existen evidencias encontradas acerca del tipo de emociones que fomentan el que una noticia sea compartida. Por un lado existen estudios que sostienen que los usuarios escogen preferentemente información que despierta emociones de tipo negativo como miedo o preocupación (Cappella, Kim, \& Albarracín, 2015) (Yang, Kahlor, \& Griffin, 2014) pero otras investigaciones sostienen que las noticias de tono positivo son más compartidas que las que provocan enfado o preocupación (Al-Rawi, 2017).

\subsection{Redes sociales y cambio climático}

Por otro lado el uso de redes sociales se ha vinculado positivamente a la participación política (Boulianne, 2015) y al compromiso cívico (Gil de Zúñiga, Jung, \& Valenzuela, 2012) de modo que se han detectado lazos entre la intensidad de uso de estas herramientas y el grado de implicación y participación en los asuntos públicos de una sociedad. De este modo, las redes sociales también tienen potencial para aumentar ese compromiso de la sociedad respecto al cambio climático (KatzKimchi \& Manosevitch, 2015). Estas plataformas pueden aumentar la atención hacia estos asuntos y visibilizar acciones de protesta (Thorson, Edgerly, Kligler-Vilenchik, Xu, \& Wang, 2016) o bien poniendo en contacto a usuarios, expertos y grupos de presión (Lee, VanDyke, \& Cummins, 2018). De hecho, el uso de las redes sociales para la información política se ha vinculado positivamente a niveles más elevados de compromiso medioambiental (Zhang \& Skoric, 2018). 
No obstante, los autores de este trabajo no han localizado estudios que analicen los factores que provocan que contenidos o noticias específicas sobre el cambio climático sean compartidos por los ciudadanos en redes sociales. La producción científica al respecto se ha centrado en el modo en qué Twitter ha cubierto eventos como la Cumbre de París (Hopke \& Hestres, 2018) y los términos y dinámicas de discusión entre usuarios sobre cambio climático en Twitter(Anderson \& Huntington, 2017) y en Youtube(Shapiro \& Park, 2018).

\subsection{Emociones y cambio climático}

Las emociones se vinculan directamente al efecto persuasivo de los mensajes (Wirz, 2018). Así las emociones son un mediador claro en los efectos y la recepción de mensajes sobre el cambio climático (Feldman \& Hart, 2016). Igualmente, los mensajes con contenido emocional pueden contribuir a concienciar el compromiso contra el cambio climático y los cambios de actitudes (Nabi, Gustafson, \& Jensen, 2018). No obstante se ha apuntado que las representaciones emocionales del cambio climático pueden reforzar el compromiso del público al respecto pero también distraer la atención lejos del propio fenómeno (Höijer, 2010).

De manera concreta se ha señalado que el miedo puede fomentar actitudes a favor del medioambiente (Hartmann, Apaolaza, D’Souza, Barrutia, \& Echebarria, 2014) y que la esperanza se relacionan con un mayor interés respecto al cambio climático (Chadwick, 2015).

No obstante, no solo el tipo de emoción sino también la intensidad de esa emoción puede influir en cómo un mensaje afecta al público. Por ejemplo, mensajes con baja carga de miedo provocan una mayor intención de adoptar actitudes pro medioambientales que los mensajes que intentan generar más miedo (Chen, 2016).

\subsection{Usos y Gratificaciones}

Por otro lado, el uso de cualquier medio no es uniforme ni universal, sino que depende de las expectativas y recompensas esperadas por cada individuo cuando se aproxima a dicho medio. Esos usos y gratificaciones esperados pueden influir en los efectos del consumo mediático (McLeod, 2000). Así la Teoría de los Usos y Gratificaciones (TUyG) se ha utilizado para explicar cambios en ciertos efectos mediáticos relacionados con el consumo y elección de medios. De este modo, diferentes expectativas llevan a diferentes patrones de usos de los medios. Así, diferentes motivaciones para el uso de las redes sociales van ligadas a diferentes actitudes a la hora de consumir o compartir noticias en estos entornos. (Choi, 2016b).

Los usos y gratificaciones más vinculados a cambios en el modo en que se comparten y consumen noticias en redes sociales son los relativos a interacción social, gestión de a propia imagen y búsqueda de información (Lee \& Ma, 2012).

Las motivaciones de tipo social, es decir, aquellas que hacen referencia a la intención de comunicarse e interactuar con otros (Whiting \& Williams, 2013), influyen en el tipo de contenido que los usuarios optan por compartir (Coppini et al., 2017). Por otro lado, los usuarios guiados por la gestión de su propia imagen, es decir por la construcción y proyección de su identidad pública (Krämer \& Winter, 2008) tienden a evitar compartir contenido de tipo negativo o controvertido (Hossain et al., 2018). Finalmente, los usos y gratificaciones vinculados a búsqueda de información, es decir a las necesidades informativas y de auto educación (Korgaonkar \& Wolin, 1999) también influyen en el consumo de noticias (Winter et al., 2016). 


\subsection{Justificación y objetivos}

Trabajos anteriores sobre el efecto de las emociones cuando se comparte información (Yang et al., 2014) ofrecen datos y evidencias limitados al contexto estadounidense. De este modo, este artículo busca aportar información en otro contexto cultural que pueda ayudar a entender mejor el papel de las emociones en la compartición y difusión de noticias.

Dado el papel relevante que desempañan las emociones en la comunicación medioambiental (Nabi et al., 2018) y la posición cada vez más relevante que las redes sociales ocupan como canal para la distribución de noticias, este trabajo intenta explicar la influencia que ejercen las emociones en la decisión de compartir o no compartir un artículo sobre el cambio climático.

A partir de este primer objetivo se plantea la pregunta de investigación PI1: “¿Qué emoción es más relevante a la hora de compartir una noticia sobre cambio climático en redes sociales?”.

De manera complementaria a esta pregunta de investigación, y a raíz de lo expuesto en el estado de la cuestión se propone también la hipótesis H1: "Las emociones negativas estarán correlacionadas con la intención de compartir una noticia en redes sociales".

Otro de los objetivos de este trabajo consiste en medir el papel moderador que ejercen otros factores sobre esta influencia emocional en la decisión de compartir una noticia acerca del cambio climático en redes sociales.

PI2. ¿Qué usos y gratificaciones perseguidos en redes sociales moderarán la influencia de las emociones a la hora de compartir una noticia sobre cambio climático?

Se han considerado las gratificaciones que, según la literatura científica, más influyen en los usuarios a la hora de compartir noticias en redes sociales (Lee \& Ma, 2012): "Gestión de la propia imagen", "Búsqueda de información" e "Interacción social". La primera de ellas, "Gestión de la propia imagen", hace referencia a aquellos aspectos de las redes sociales que permiten a los usuarios construir y proyectar una presentación de su persona (Krämer; Winter, 2008). "Búsqueda de información", por su parte, implica que los usuarios eligen el medio para su propia formación y para satisfacer necesidades informativas (Korgaonkar; Wolin, 1999). Por último, "Interacción social", se refiere a la intención de comunicarse e interactuar con otros (Whiting; Williams, 2013).

Por otro lado, los efectos de la exposición a medios y los patrones de consumo de información están condicionados por el conjunto de hábitos y patrones propio de cada usuario (LaRose \& Eastin, 2004). En este sentido, la audiencia no realiza un nuevo ejercicio racional y consciente cada vez que quiere consultar noticias o contenidos mediáticos, sino que se basa en un repertorio prefijado de plataformas tecnológicas, medios y cabeceras concretas. Así, por ejemplo, los ciudadanos pueden optar por recibir sus noticias a través de medios tradicionales o buscar ese contenido en medios digitales. Esta elección afecta al resto de su implicación con las noticias (compartir o marcar como "me gusta"). De hecho, aquellos usuarios que dependen más de medios digitales han demostrado ser más activos en este tipo de hábitos (Choi, 2016b; Karnowski, Leonhard \& Kümpel, 2018).

Como ya se ha mencionado, las redes sociales no solo sirven como un vehículo de consumo de información sino también representan un canal secundario de distribución de noticias. En este sentido Choi (2016a) sugiere que los hábitos de los usuarios en redes sociales deben diferenciarse entre internalización de noticias (cómo los usuarios reciben noticias en redes sociales) y externalización de noticias (cómo los usuarios comparten noticias en redes sociales). 
Así pues, dado el papel que ejercen los hábitos mediáticos se plantea la pregunta de investigación PI3, “Qué hábitos de consumo informativo en redes sociales moderarán la influencia de las emociones a la hora de compartir una noticia sobre cambio climático?".

Por otro lado, en el campo de la comunicación política se ha comprobado que las actitudes y opiniones previas pueden influir y amortiguar las reacciones emocionales provocadas por los mensajes (Feldman \& Hart, 2016; Hasell \& Weeks, 2016). Por ello se quiere comprobar si estas actitudes previas hacia el cambio climático pueden moderar la influencia emocional a la hora de compartir una noticia en redes sociales, de modo que se plantea la siguiente pregunta de investigación: PI4. ¿Qué actitudes previas hacia el cambio climático moderarán la influencia de las emociones a la hora de compartir una noticia en redes sociales sobre este asunto?

De manera similar se ha señalado que el conocimiento previo sobre un tema ejerce un papel decisivo a la hora de leer información que los usuarios encuentran incidentalmente en redes sociales (Karnowski et al., 2017). Del mismo modo el grado de conocimiento previo sobre el tema es uno de los principales factores que provocan la discusión y comentario de noticias sobre cambio climático (Taddicken \& Reif, 2016). Por ello se propone la hipótesis H2: "El conocimiento previo sobre el cambio climático moderará la influencia de las emociones sobre la intención de compartir una noticia en redes sociales sobre este asunto". De modo adicional se plantea una hipótesis complementaria dirigida no tanto al grado de conocimiento previo, sino al interés existente hacia el tema. Así se propone la hipótesis H3: "El grado de preocupación previo sobre el cambio climático moderará la influencia de las emociones sobre la intención de compartir una noticia en redes sociales sobre este asunto".

\section{Metodología}

Se envió un cuestionario online a una muestra $(n=96)$ de estudiantes universitarios de todas las universidades (públicas y privadas) de la Comunidad de Madrid, procedentes de distintos grados para evitar un perfil homogéneo en lo relativo a su formación. Una empresa de estudios sociológicos se encargó de reclutar de manera aleatoria a los participantes, que fueron invitados a formar parte del estudio a cambio de un cheque regalo de $25 €$ para unos grandes almacenes. Las respuestas se recogieron entre el 5 de mayo y el 3 de julio de 2017.

El tamaño de la muestra va en línea de estudios similares que analizan el modo en que determinados factores influyen en la percepción de una noticia o de un asunto particular (ver por ejemplo: Da Silva \& Pereira, 2017; Gerber et al., 2017; Penney \& Abbott, 2015).

La edad media de los participantes fue de 20,23 años (desviación estándar $=3$ ). El 61,46\% de los participantes fueron mujeres y el 38,54\% hombres. Los participantes respondieron de manera online un cuestionario autoadministrado.

El universo de estudio se centró en jóvenes adultos puesto que se trata de un sector demográfico especialmente activo a la hora de compartir y consumir noticias online (Antunovic, Parsons, \& Cooke, 2018). Esto permite esperar que en el futuro sus hábitos marque y desarrollen la evolución de las tendencias a la hora de compartir noticias (Bobkowski, 2015).

A continuación, se detallan las distintas escalas y medidas empleadas en el cuestionario. Se ha calculado la coherencia interna mediante el alfa de Cronbach, indicado en la tabla correspondiente a cada constructo con el símbolo $\alpha$ para medir los hábitos de consumo informativo en redes sociales de los participantes se emplearon los constructos "Internalización de noticias" (es decir, la frecuencia 
con que los usuarios reciben y leen noticias) y "Externalización de noticias" (referido a la frecuencia con la que comparten noticias) y las respectivas escalas definidas por Choi (2016a). Para ello se obtuvo el sumatorio de las respuestas a una escala Likert (1="Nunca", 7= "Todos los días") como se muestra en la tabla 1.

Tabla 1. Medidas de los hábitos de consumo informativo.

\begin{tabular}{|l|l|}
\hline \multirow{4}{*}{$\begin{array}{l}\text { Internalización de noticias } \\
(\alpha=0,687)\end{array}$} & Leo noticias en redes sociales \\
\cline { 2 - 2 } & $\begin{array}{l}\text { Recibo enlaces a noticias (mediante cuentas de medios a los que } \\
\text { sigo) }\end{array}$ \\
\cline { 2 - 2 } & $\begin{array}{l}\text { Recibo enlaces a noticias de mis contactos (personales, no } \\
\text { cuentas de medios de comunicación) }\end{array}$ \\
\hline $\begin{array}{l}\text { Externalización de noticias } \\
(\alpha=0,76)\end{array}$ & Publico noticias en redes sociales \\
\cline { 2 - 2 } & Comparto enlaces a noticias con mis contactos \\
\hline
\end{tabular}

Fuente: Choi \& Lee, 2015.

Siguiendo a Hyun \& Kim (2015), el grado del conocimiento previo sobre cambio climático se ha calculado como el sumatorio de respuestas correctas a un conjunto de preguntas donde los usuarios debían elegir entre "verdadero" y "falso". Cada respuesta correcta se puntuó con uno, mientras que las respuestas incorrectas no sumaban ningún punto (media $=1,54$, desviación estándar $=0,87$ ) (ver tabla 2).

Tabla 2. Preguntas sobre conocimiento del cambio climático.

\begin{tabular}{l}
\hline $\begin{array}{l}\text { Los primeros registros históricos de la temperatura datan de unos } 150 \text { años. Desde entonces, los } \\
\text { registros no han hecho más que aumentar (Verdadero) }\end{array}$ \\
\hline El CO2 es el único gas industrial responsable del efecto invernadero (Falso) \\
\hline Estados Unidos ha ratificado el protocolo de Kioto (Falso) \\
\hline $\begin{array}{l}\text { Cada país adherido al protocolo de Kioto se compromete a reducir sus emisiones de CO2 en un } 5 \% \\
\text { (Falso) }\end{array}$
\end{tabular}

Fuente: Hyun \& Kim (2015).

La preocupación general sobre el cambio climático se evaluó a través de una única pregunta tipo Likert (1="Nada preocupado", 7="Muy preocupado"). En la tabla 3 se muestran las preguntas empleadas para medir las distintas actitudes de los usuarios respectos al cambio climático.

Tabla 3. Preguntas sobre actitudes respecto al cambio climático.

\begin{tabular}{|c|c|c|}
\hline & Media & $\begin{array}{l}\text { Desviación } \\
\text { estándar }\end{array}$ \\
\hline El cambio climático es consecuencia de la acción del hombre & 5,93 & 1,43 \\
\hline $\begin{array}{l}\text { El cambio climático es un fenómeno natural, independiente de la acción } \\
\text { del hombre }\end{array}$ & 2,18 & 1,3 \\
\hline $\begin{array}{l}\text { El cambio climático puede detenerse si gobiernos y ciudadanos toman } \\
\text { las medidas oportunas }\end{array}$ & 5,71 & 1,3 \\
\hline
\end{tabular}

Fuente: elaboración propia. 
RLCS, Revista Latina de Comunicación Social, 75, 245-269

[Investigación] DOI: 10.4185/RLCS-2020-1425| ISSN 1138-5820 | Año 2020

Los usos y gratificaciones considerados en el estudio se construyeron como el sumatorio de preguntas tipo Likert (1="Nada de acuerdo", 7="Totalmente de acuerdo") independientes mostradas en la tabla 4 y adaptadas de (Gao \& Feng, 2016).

Tabla 4. Medidas para los usos y gratificaciones de redes sociales.

\begin{tabular}{|c|c|}
\hline \multirow{6}{*}{$\begin{array}{l}\text { Búsqueda de información } \\
(\alpha=0,9)\end{array}$} & $\begin{array}{l}\text { Puedo acceder rápida y fácilmente a una gran cantidad de } \\
\text { información }\end{array}$ \\
\hline & Puedo conseguir información útil \\
\hline & Puedo consultar información a muy bajo coste \\
\hline & Puedo conseguir información que me interesa \\
\hline & $\begin{array}{l}\text { Puedo usar las redes sociales para conservar información que } \\
\text { puedo utilizar en el futuro }\end{array}$ \\
\hline & Puedo aprender mucho \\
\hline \multirow{5}{*}{$\begin{array}{l}\text { Interacción social } \\
(\alpha=0,912)\end{array}$} & Puedo conseguir información sobre mis amigos \\
\hline & Puedo comunicarme e interactuar con mis amigos \\
\hline & Puedo mostrar preocupación y apoyo a mis amigos \\
\hline & Puedo conseguir opinión y consejos de mis amigos \\
\hline & Puedo expresar mis ideas y dar consejos a mis amigos \\
\hline \multirow{6}{*}{$\begin{array}{l}\text { Gestión de la propia imagen } \\
(\alpha=0,91)\end{array}$} & Puedo conocer gente nueva \\
\hline & Puedo sentirme conectado \\
\hline & Me ayudan a gustarle a otros \\
\hline & Puedo hacer que otros piensen que soy amigable \\
\hline & Puedo hacer que otros piensen que soy sociable \\
\hline & $\begin{array}{l}\text { Puedo hacer que otros piensen que soy competente y capaz de } \\
\text { hacer cosas }\end{array}$ \\
\hline
\end{tabular}

Fuente: Gao \& Feng, 2016.

Una vez respondidas estas preguntas el formulario pedía a los participantes que leyeran una noticia sobre los efectos del cambio climático. El texto y las imágenes del artículo (reproducidos de: La Vanguardia, 2017) no incluían referencias a su fuente, ni a ningún otro contexto. Una vez leída la noticia, los participantes tuvieron que medir su respuesta emocional a través de una serie de preguntas tipo Likert mostradas en la tabla $5(1=$ "No he sentido en absoluto esta emoción", 7 ="He sentido mucho esta emoción").

Tabla 5. Emociones.

\begin{tabular}{|r|r|r|}
\hline $\begin{array}{l}\text { ¿Hasta qué punto ha sentido las siguientes emociones al leer la } \\
\text { noticia? }\end{array}$ & Media & Desviación estándar \\
\hline Miedo & 3,76 & 1,88 \\
\hline Sospecha & 3,2 & 1,66 \\
\hline Rabia & 4,55 & 1,86 \\
\hline Confusión & 2,75 & 1,68 \\
\hline Tristeza & 4,47 & 1,84 \\
\hline
\end{tabular}

Fuente: elaboración propia. 
A partir de estas medidas se elaboró un índice de intensidad de las emociones negativas que se obtuvo con la suma de la puntuación obtenida en miedo, rabia, tristeza y preocupación (media $=18.08$, desviación estándar=6.03).

Por último, los participantes tuvieron que responder a la pregunta: “¿Compartiría la noticia que ha leído en redes sociales?". El formulario solo permitía responder "Sí" o "No".

\subsection{Tratamiento estadístico}

El modelo estadístico empleado fue:

- Modelo de regresión lineal simple y múltiple cuando la variable dependiente fue continúa. El coeficiente mostrado en las tablas será un coeficiente lineal.

- Modelo de regresión logística simple y múltiple cuando la variable dependiente fue binaria. El coeficiente mostrado en las tablas será el odds ratio.

- Modelo de regresión ordinal simple y múltiple cuando la variable dependiente fuese discreta con más de dos categorías con una relación de orden entre ellas. El coeficiente mostrado en las tablas será el odds ratio.

Estos modelos son habituales en otros estudios similares tanto en Comunicación (Chyi \& Yang, 2009; Stempel, Hargrove, \& Stempel, 2007), como en otras disciplinas, (Kaufman, Dwyer, Land, Klein \& Park, 2018; Kirk, Lee, Ang \& Lee, 2015) destinados a medir relaciones entre percepciones, evaluaciones de mensajes y otros constructos o aspectos.

El diseño elegido para las tablas fue el siguiente: la primera fila hace referencia al modelo lineal simple, es decir, sin ninguna variable que pueda mediar o moderar su efecto y se muestra el coeficiente (lineal o odds ratio) y su intervalo de confianza al 95\%. El resto de las filas tienen en cuenta cada una de las variables que pueden mediar o moderar el efecto. Por tanto, el coeficiente mostrado hacer referencia a la variable independiente principal pero alterado por la presencia de la variable mediadora correspondiente.

Para determinar si una variable es moderadora o mediadora se tuvo en consideración el criterio bioestadístico de la Universidad de Penn State en sus cursos de bioestadística: si el coeficiente cuando se tiene en cuenta una tercera variable varía en más de un $10 \%$ con respecto al coeficiente asociado al modelo sin dicha tercera variable, ésta será considerada mediadora o moderadora, en caso contrario no.

La fórmula empleada para calcular el cambio porcentual utilizada fue:

$$
\text { Variación } \%=\left(\frac{\mid \text { coe } f_{\text {sinvarmoderadora }}-\operatorname{coef}_{\text {convarmoderadora }} \mid}{\text { coef }_{\text {sinvarmoderadora }}}\right) \times 100
$$

De este modo, todas aquellas variables que son mediadoras o moderadoras se resaltan en negrita en cada una de las tablas. Además, en el caso de que el coeficiente sea estadísticamente significativo, se indica con un asterisco sobre el propio coeficiente.

\section{Resultados}

\subsection{Influencia de las emociones en la intención de compartir la noticia}

El odds ratio asociado al miedo, proporcionado por el modelo de regresión logística simple alcanzó la significación estadística, siendo de 1,2892 con un intervalo de confianza al $95 \%$ de $(1,0311$, 
1,6309) (ver gráfico 1). Esto indica que un mayor nivel del miedo se traduce en una mayor probabilidad a la hora de compartir la noticia. Concretamente de acuerdo al odds ratio, un aumento en una unidad en dicho nivel hace que el aumento de la probabilidad de compartir la noticia aumente en casi un $29 \%$. Aunque como se puede ver en el gráfico, donde existe una mayor probabilidad de compartir la noticia es entre los individuos con un mayor grado en dicha preocupación. Aunque en la categoría inferior del nivel de miedo, la situación sea contraria, debido quizá a esa falta de miedo.

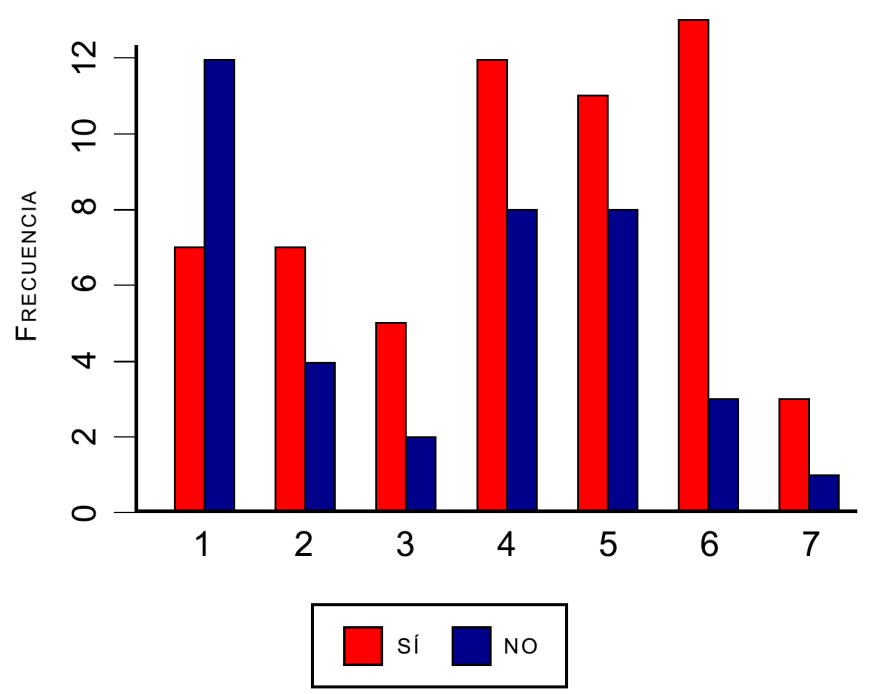

Gráfico 1: frecuencia de participantes que compartirían la noticia en función del miedo percibido.

Fuente: elaboración propia.

El odds ratio asociado a la sospecha, proporcionado por el modelo de regresión logística simple no alcanzó la significación estadística, siendo de 1,0932 con un intervalo de confianza al 95\% de $(0,8525,1,4127)$. Esto indica que un mayor nivel de sospecha no se traduce en una mayor probabilidad a la hora de compartir la noticia.

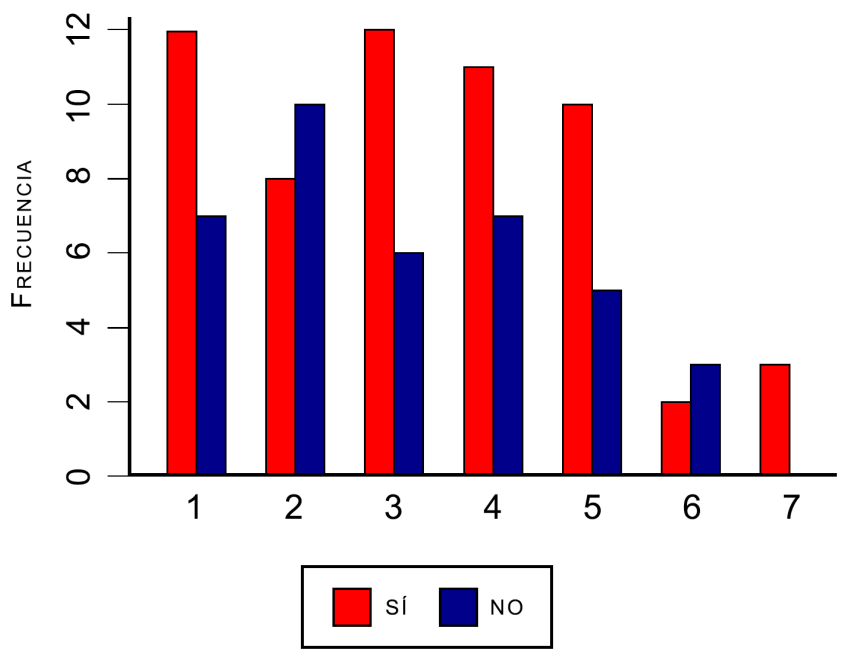

Gráfico 2: frecuencia de participantes que compartirían la noticia en función de la sospecha percibida.

Fuente: elaboración propia. 
El odds ratio asociado a la rabia, proporcionado por el modelo de regresión logística simple alcanzó la significación estadística, siendo de 1,35 con un intervalo de confianza al 95\% de $(1,0755,1,7217)$. Esto indica que un mayor nivel de rabia se traduce en una mayor probabilidad a la hora de compartir la noticia. Concretamente de acuerdo al odds ratio, un aumento en una unidad en dicho nivel hace que el aumento de la probabilidad de compartir la noticia aumente en casi un $35 \%$. Aunque como se puede ver en el gráfico, donde existe realmente una mayor probabilidad de compartir la noticia es entre los individuos con un mayor grado en dicha emoción.

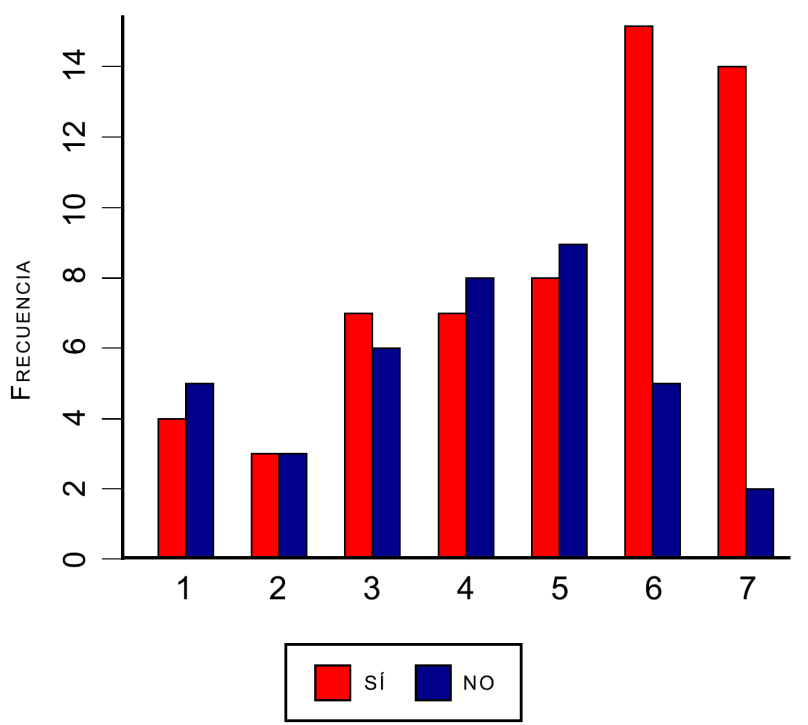

Gráfico 3: frecuencia de participantes que compartirían la noticia en función de la rabia percibida.

Fuente: elaboración propia.

El odds ratio asociado al nivel de confusión, proporcionado por el modelo de regresión logística simple no alcanzó la significación estadística, siendo de 1,0565 con un intervalo de confianza al 95\% de $(0,827,1,3601)$. Esto indica que un mayor nivel de confusión no se traduce en una mayor probabilidad a la hora de compartir la noticia.

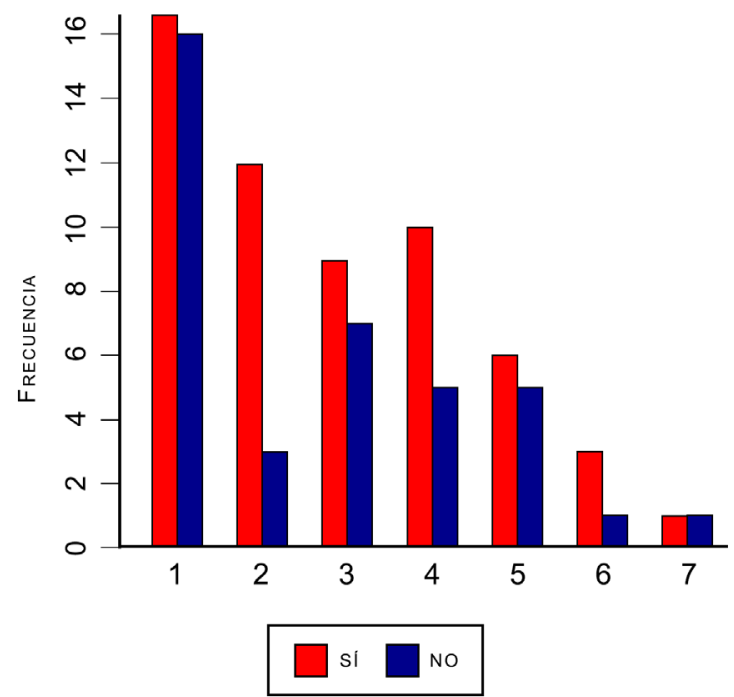

Gráfico 4: frecuencia de participantes que compartirían la noticia en función de la confusión percibida.

Fuente: elaboración propia. 
El odds ratio asociado al nivel de tristeza, proporcionado por el modelo de regresión logística simple no alcanzó la significación estadística, siendo de 1,1666 con un intervalo de confianza al 95\% de $(0,9324,1,47)$. Esto indica que un mayor nivel de tristeza no se traduce en una mayor probabilidad a la hora de compartir la noticia. Aunque como se puede ver en el gráfico en niveles de tristeza de 6 y de 7 la diferencia de compartir la noticia es más notable.

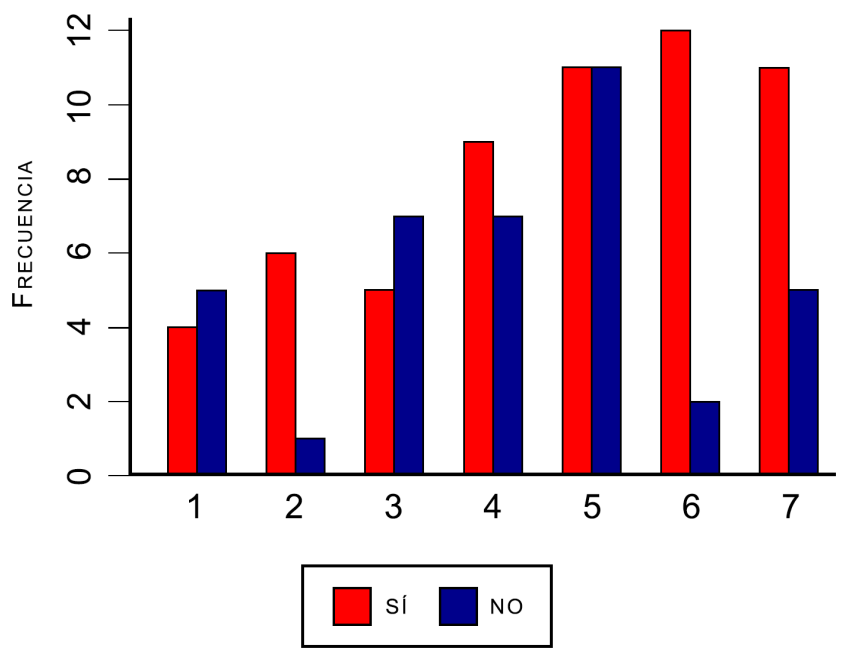

Gráfico 5: frecuencia de participantes que compartirían la noticia en función de la tristeza percibida.

Fuente: elaboración propia.

El odds ratio asociado al nivel de preocupación, proporcionado por el modelo de regresión logística simple no alcanzó la significación estadística, siendo de 1,2001 con un intervalo de confianza al 95\% de $(0,9467,1,5362)$. Esto indica que un mayor nivel de preocupación no se traduce en una mayor probabilidad a la hora de compartir la noticia. Aunque como se puede ver en el gráfico, en niveles de preocupación de 6 y de 7 la diferencia de compartir la noticia es más notable.

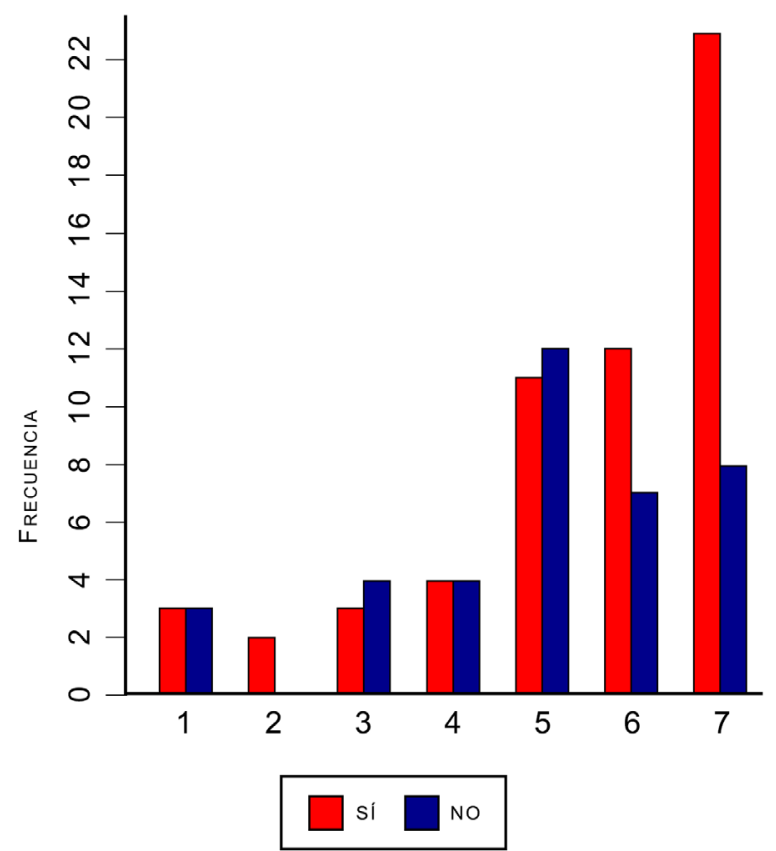

Gráfico 6: frecuencia de participantes que compartirían la noticia en función de la preocupación percibida.

Fuente: elaboración propia. 
El odds ratio asociado a la influencia de emociones negativas, proporcionado por el modelo de regresión logística simple alcanzó la significación estadística, siendo de 1,0872 con un intervalo de confianza al $95 \%$ de $(1,0137,1,172)$. Esto indica que un incremento de una unidad en la influencia de emociones negativas, se traduce en una mayor probabilidad de compartir la noticia. Concretamente, de acuerdo al gráfico y al odds ratio, el aumento de la probabilidad de compartir la noticia aumenta en un $8,7 \%$.

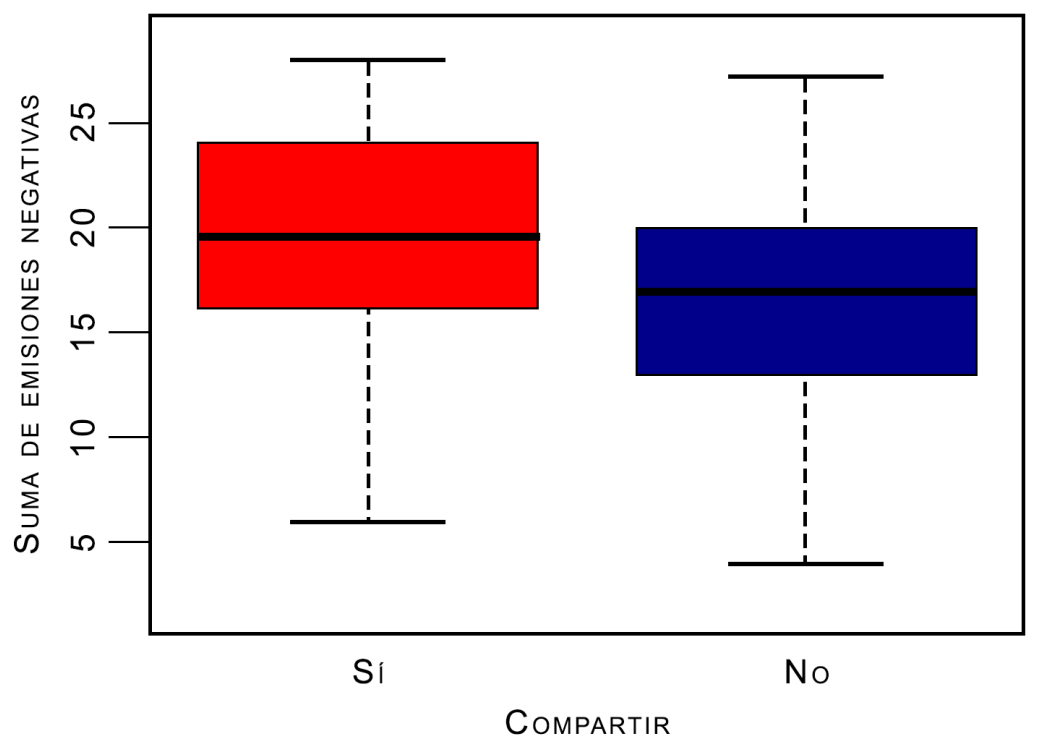

Gráfico 7: frecuencia de participantes que compartirían la noticia en función de las emociones negativas percibidas.

Fuente: elaboración propia.

\subsection{Papel moderador de los usos y gratificaciones perseguidos}

Se puede considerar variable moderadora la búsqueda de información en los casos de "Rabia", "Sospecha" y "Preocupación". En estas ocasiones se produce un cambio mayor del 10\% en el odds ratio asociado a que la noticia sea compartida (ver tabla 6).

Tabla 6. Papel moderador de los usos y gratificaciones en la influencia de las emociones percibidas y la intención de compartir la noticia.

\begin{tabular}{|c|c|c|c|c|l|}
\hline Emoción & $\begin{array}{c}\text { Regresión } \\
\text { Ordinal }\end{array}$ & $\begin{array}{c}\mathbf{9 5 \%} \text { CI } \\
\text { Inferior }\end{array}$ & $\begin{array}{c}\mathbf{9 5 \%} \text { CI } \\
\text { Superior }\end{array}$ & $\begin{array}{c}\text { \% } \\
\text { Cambio }\end{array}$ & Variable moderadora \\
\hline \multirow{4}{*}{ Miedo } & 1,2892 & 1,0311 & 1,6309 & & \\
\cline { 2 - 7 } & 1,3694 & 1,0828 & 1,7613 & 6,2209 & Búsqueda de información \\
\cline { 2 - 6 } & 1,3251 & 1,0532 & 1,691 & 2,7847 & Interacción social \\
\cline { 2 - 6 } & 1,2901 & 1,0317 & 1,6322 & 0,0698 & $\begin{array}{l}\text { Gestión de la propia } \\
\text { imagen }\end{array}$ \\
\hline \multirow{4}{*}{ Sospecha } & 1,0932 & 0,8525 & 1,4127 & & \\
\cline { 2 - 6 } & 1,1436 & 0,8842 & 1,4976 & 4,6103 & Búsqueda de información \\
\cline { 2 - 6 } & 1,0792 & 0,8382 & 1,3983 & 1,2806 & Interacción social \\
\cline { 2 - 6 } & 1,0923 & 0,8515 & 1,4118 & 0,0823 & $\begin{array}{l}\text { Gestión de la propia } \\
\text { imagen }\end{array}$ \\
\hline
\end{tabular}


RLCS, Revista Latina de Comunicación Social, 75, 245-269

[Investigación] DOI: 10.4185/RLCS-2020-1425| ISSN 1138-5820| Año 2020

\begin{tabular}{|c|c|c|c|c|c|}
\hline \multirow{4}{*}{ Rabia } & $1,35^{*}$ & 1,0755 & 1,7217 & & \\
\hline & $1,5954 *$ & 1,2209 & 2,1544 & 18,1778 & Búsqueda de información \\
\hline & $1,3986^{*}$ & 1,1042 & 1,8047 & 3,6 & Interacción social \\
\hline & $1,3495^{*}$ & 1,075 & 1,7212 & 0,037 & $\begin{array}{l}\text { Gestión de la propia } \\
\text { imagen }\end{array}$ \\
\hline \multirow{4}{*}{ Confusión } & 1,0565 & 0,827 & 1,3601 & & \\
\hline & 1,0816 & 0,8434 & 1,3998 & 2,3758 & Búsqueda de información \\
\hline & 1,0711 & 0,8365 & 1,3811 & 1,3819 & Interacción social \\
\hline & 1,0549 & 0,8248 & 1,359 & 0,1514 & $\begin{array}{l}\text { Gestión de la propia } \\
\text { imagen }\end{array}$ \\
\hline \multirow{4}{*}{ Tristeza } & 1,1666 & 0,9324 & 1,47 & & \\
\hline & 1,3082 & 1,021 & 1,7067 & 12,1378 & Búsqueda de información \\
\hline & 1,2826 & 1,005 & 1,6622 & 9,9434 & Interacción social \\
\hline & 1,1657 & 0,9308 & 1,4703 & 0,0771 & $\begin{array}{l}\text { Gestión de la propia } \\
\text { imagen }\end{array}$ \\
\hline \multirow{4}{*}{ Preocupación } & 1,2001 & 0,9467 & 1,5362 & & \\
\hline & $1,4072 *$ & 1,0686 & 1,8988 & 17,2569 & Búsqueda de información \\
\hline & $1,2963 *$ & 1,0048 & 1,7021 & 8,016 & Interacción social \\
\hline & 1,1995 & 0,945 & 1,5372 & 0,05 & $\begin{array}{l}\text { Gestión de la propia } \\
\text { imagen }\end{array}$ \\
\hline \multirow{4}{*}{$\begin{array}{c}\text { Intensidad de } \\
\text { las emociones } \\
\text { negativas }\end{array}$} & 1,0872 & 1,0137 & 1,172 & & \\
\hline & $1,1509^{*}$ & 1,0587 & 1,2637 & 5,8591 & Búsqueda de información \\
\hline & $1,1141^{*}$ & 1,0332 & 1,2095 & 2,4742 & Interacción social \\
\hline & $1,0871^{*}$ & 1,0135 & 1,1729 & 0,009198 & $\begin{array}{l}\text { Gestión de la propia } \\
\text { imagen }\end{array}$ \\
\hline
\end{tabular}

Fuente: Elaboración propia.

\subsection{Papel moderador de la internalización y externalización de noticias}

Se puede considerar variable moderadora la internalización de la noticia. Se debe a que produce un cambio mayor del $10 \%$ en el odds ratio asociado a que la noticia sea compartida en los casos de "Rabia", "Tristeza" y "Preocupación" (ver tabla 7).

Tabla 7. Papel moderador de la internalización y externalización en la influencia de las emociones percibidas y la intención de compartir la noticia.

\begin{tabular}{|c|c|c|c|c|c|}
\hline Emoción & $\begin{array}{c}\text { Regresión } \\
\text { Ordinal }\end{array}$ & $\begin{array}{l}95 \% \text { CI } \\
\text { Inferior }\end{array}$ & $\begin{array}{l}95 \% \text { CI } \\
\text { Superior }\end{array}$ & $\begin{array}{c}\% \\
\text { Cambio }\end{array}$ & Variable moderadora \\
\hline \multirow{3}{*}{ Miedo } & $1,2892 *$ & 1,0311 & 1,6309 & & \\
\hline & $1,3872 *$ & 1,0888 & 1,8056 & 7,6016 & Internalización noticias \\
\hline & $1,306^{*}$ & 1,033 & 1,674 & 1,3031 & Externalización noticias \\
\hline \multirow{3}{*}{ Sospecha } & 1,0932 & 0,8525 & 1,4127 & & \\
\hline & 1,1062 & 0,854 & 1,4462 & 1,1892 & Internalización noticias \\
\hline & 1,0943 & 0,8531 & 1,4146 & 0,1006 & Externalización noticias \\
\hline \multirow{3}{*}{ Rabia } & $1,35^{*}$ & 1,0755 & 1,7217 & & \\
\hline & $1,6263^{*}$ & 1,2343 & 2,2259 & 20,4667 & Internalización noticias \\
\hline & $1,3517 *$ & 1,0739 & 1,7283 & 0,1259 & Externalización noticias \\
\hline \multirow{3}{*}{ Confusión } & 1,0565 & 0,827 & 1,3601 & & \\
\hline & 1,0864 & 0,8444 & 1,4124 & 2,8301 & Internalización noticias \\
\hline & 1,0494 & 0,8164 & 1,3586 & 0,672 & Externalización noticias \\
\hline
\end{tabular}


RLCS, Revista Latina de Comunicación Social, 75, 245-269

[Investigación] DOI: 10.4185/RLCS-2020-1425| ISSN 1138-5820 | Año 2020

\begin{tabular}{|c|c|c|c|c|l|}
\hline \multirow{4}{*}{ Tristeza } & 1,1666 & 0,9324 & 1,47 & \multicolumn{2}{|c|}{} \\
\cline { 2 - 6 } & 1,3202 & 1,0263 & 1,7339 & $\mathbf{1 3 , 1 6 6 5}$ & Internalización noticias \\
\cline { 2 - 6 } & 1,1746 & 0,9242 & 1,5051 & 0,6858 & Externalización noticias \\
\hline \multirow{3}{*}{\begin{tabular}{c} 
Preocupación \\
\cline { 2 - 6 }
\end{tabular}} & 1,2001 & 0,9467 & 1,5362 & \multicolumn{2}{|c|}{} \\
\cline { 2 - 6 } & 1,3815 & 1,0562 & 1,8444 & $\mathbf{1 5 , 1 1 5 4}$ & Internalización noticias \\
\hline \multirow{2}{*}{$\begin{array}{c}\text { Intensidad de } \\
\text { las emociones } \\
\text { negativas }\end{array}$} & 1,1982 & 0,9417 & 1,5392 & 0,1583 & Externalización noticias \\
\cline { 2 - 6 } & $1,1542^{*}$ & 1,0601 & 1,2704 & 6,1626 & Internalización noticias \\
\cline { 2 - 6 } & $1,0922^{*}$ & 1,0147 & 1,1822 & 0,4599 & Externalización noticias \\
\hline
\end{tabular}

Fuente: elaboración propia.

\subsection{Papel moderador de interés, conocimiento y actitudes previos}

Se puede considerar variable moderadora la preocupación por el cambio climático. Se debe a que produce un cambio mayor del $10 \%$ en el odds ratio asociado a que la noticia sea compartida (ver tabla 8).

Tabla 8. Papel moderador del interés, conocimiento y actitudes previos en la influencia de las emociones percibidas y la intención de compartir la noticia.

\begin{tabular}{|c|c|c|c|c|c|}
\hline Emoción & $\begin{array}{c}\text { Regresión } \\
\text { Ordinal }\end{array}$ & $\begin{array}{l}95 \% \mathrm{CI} \\
\text { Inferior }\end{array}$ & $\begin{array}{l}95 \% \mathrm{CI} \\
\text { Superior }\end{array}$ & $\begin{array}{c}\% \% \\
\text { Cambio }\end{array}$ & Variable moderadora \\
\hline \multirow{6}{*}{ Miedo } & $1,2892 *$ & 1,0311 & 1,6309 & & \\
\hline & 1,2007 & 0,9435 & 1,537 & 6,8647 & $\begin{array}{l}\text { Preocupación por el } \\
\text { cambio climático }\end{array}$ \\
\hline & $1,3376^{*}$ & 1,0576 & 1,7194 & 3,7543 & $\begin{array}{l}\text { Fenómeno provocado por } \\
\text { el hombre }\end{array}$ \\
\hline & $1,2947 *$ & 1,0333 & 1,6417 & 0,4266 & Fenómeno natural \\
\hline & $1,2775^{*}$ & 1,0199 & 1,6181 & 0,9075 & Frenar el cambio climático \\
\hline & 1,3214 & 1,0503 & 1,0503 & 2,4977 & $\begin{array}{l}\text { Conocimiento previo por } \\
\text { el tema }\end{array}$ \\
\hline \multirow{6}{*}{ Sospecha } & 1,0932 & 0,8525 & 1,4127 & & \\
\hline & 0,9897 & 0,7525 & 1,3016 & 9,4676 & $\begin{array}{l}\text { Preocupación por el } \\
\text { cambio climático }\end{array}$ \\
\hline & 1,1036 & 0,8582 & 1,4322 & 0,9513 & $\begin{array}{l}\text { Fenómeno provocado por } \\
\text { el hombre }\end{array}$ \\
\hline & 1,0945 & 0,8527 & 1,4153 & 0,1189 & Fenómeno natural \\
\hline & 1,0838 & 0,843 & 1,403 & 0,8599 & Frenar el cambio climático \\
\hline & 1,1157 & 0,8674 & 0,8674 & 2,05818 & $\begin{array}{l}\text { Conocimiento previo por } \\
\text { el tema }\end{array}$ \\
\hline \multirow{6}{*}{ Rabia } & 1,35 & 1,0755 & 1,7217 & & \\
\hline & 1,2038 & 0,9246 & 1,5744 & 10,8296 & $\begin{array}{l}\text { Preocupación por el } \\
\text { cambio climático }\end{array}$ \\
\hline & 1,5066 & 1,1546 & 2,0314 & $1,1,6$ & $\begin{array}{l}\text { Fenómeno provocado por } \\
\text { el hombre }\end{array}$ \\
\hline & 1,3647 & 1,0819 & 1,7524 & 1,0889 & Fenómeno natural \\
\hline & 1,3371 & 1,0584 & 1,7155 & 0,9556 & Frenar el cambio climático \\
\hline & 1,4113 & 1,1136 & 1,1136 & 4,5407 & $\begin{array}{l}\text { Conocimiento previo por } \\
\text { el tema }\end{array}$ \\
\hline Confusión & 1,0565 & 0,827 & 1,3601 & & \\
\hline
\end{tabular}


RLCS, Revista Latina de Comunicación Social, 75, 245-269

[Investigación] DOI: 10.4185/RLCS-2020-1425| ISSN 1138-5820| Año 2020

\begin{tabular}{|c|c|c|c|c|c|}
\hline & 1,0646 & 0,8192 & 1,3933 & 0,7667 & $\begin{array}{l}\text { Preocupación por el } \\
\text { cambio climático }\end{array}$ \\
\hline & 1,0532 & 0,8234 & 1,3569 & 0,3124 & $\begin{array}{l}\text { Fenómeno provocado por } \\
\text { el hombre }\end{array}$ \\
\hline & 1,0586 & 0,8265 & 1,366 & 0,1988 & Fenómeno natural \\
\hline & 1,0686 & 0,8346 & 1,3806 & 1,1453 & Frenar el cambio climático \\
\hline & 1,0975 & 0,8503 & 1,4327 & 3,8807 & $\begin{array}{l}\text { Conocimiento previo por } \\
\text { el tema }\end{array}$ \\
\hline \multirow{6}{*}{ Tristeza } & 1,1666 & 0,9324 & 1,47 & & \\
\hline & 1,0304 & 0,7918 & 1,3293 & 11,675 & $\begin{array}{l}\text { Preocupación por el } \\
\text { cambio climático }\end{array}$ \\
\hline & 1,2336 & 0,9635 & 1,5987 & 5,7432 & $\begin{array}{l}\text { Fenómeno provocado por } \\
\text { el hombre }\end{array}$ \\
\hline & 1,1716 & 0,9329 & 1,4822 & 0,4286 & Fenómeno natural \\
\hline & 1,1431 & 0,9027 & 1,4547 & 2,0144 & Frenar el cambio climático \\
\hline & 1,1931 & 0,9486 & 0,9486 & 2,2716 & $\begin{array}{l}\text { Conocimiento previo por } \\
\text { el tema }\end{array}$ \\
\hline \multirow{7}{*}{ Preocupación } & 1,2001 & 0,9467 & 1,5362 & & \\
\hline & 1,1579 & 0,9024 & 1,4944 & 3,5164 & $\begin{array}{l}\text { Interés por la información } \\
\text { científica }\end{array}$ \\
\hline & 1,0521 & 0,7976 & 1,3787 & 12,3323 & $\begin{array}{l}\text { Preocupación por el } \\
\text { cambio climático }\end{array}$ \\
\hline & 1,2794 & 0,9829 & 1,6975 & 6,6078 & $\begin{array}{l}\text { Fenómeno provocado por } \\
\text { el hombre }\end{array}$ \\
\hline & 1,2072 & 0,948 & 1,5549 & 0,5916 & Fenómeno natural \\
\hline & 1,1774 & 0,9192 & 1,5202 & 1,8915 & Frenar el cambio climático \\
\hline & 1,2349 & 0,9597 & 0,9597 & 2,90 & $\begin{array}{l}\text { Conocimiento previo por } \\
\text { el tema }\end{array}$ \\
\hline \multirow{7}{*}{$\begin{array}{c}\text { Intensidad de } \\
\text { las emociones } \\
\text { negativas }\end{array}$} & $1,0872 *$ & 1,0137 & 1,172 & & \\
\hline & $1,0795^{*}$ & 1,0041 & 1,1658 & 0,7082 & $\begin{array}{l}\text { Interés por la información } \\
\text { científica }\end{array}$ \\
\hline & 1,0474 & 0,9651 & 1,138 & 3,6608 & $\begin{array}{l}\text { Preocupación por el } \\
\text { cambio climático }\end{array}$ \\
\hline & $1,1272 *$ & 1,0377 & 1,2357 & 3,6792 & $\begin{array}{l}\text { Fenómeno provocado por } \\
\text { el hombre }\end{array}$ \\
\hline & $1,0914^{*}$ & 1,0157 & 1,1794 & 0,3863 & Fenómeno natural \\
\hline & $1,0839 *$ & 1,0076 & 1,1714 & 0,3035 & Frenar el cambio climático \\
\hline & 1,0997 & 1,0226 & 1,189 & 1,1497 & $\begin{array}{l}\text { Conocimiento previo por } \\
\text { el tema }\end{array}$ \\
\hline
\end{tabular}

Fuente: elaboración propia.

\section{Discusión y conclusiones}

Los datos obtenidos permiten contestar las preguntas de investigación enunciadas y contrastar las hipótesis planteadas. Así, respecto a la PI1 “¿Qué emoción es más relevante a la hora de compartir una noticia sobre cambio climático en redes sociales?" se puede señalar que el miedo y la rabia son las emociones que más han fomentado el que una noticia se comparta (ver gráficos 1 y 3 ). Asimismo, también se puede dar por verificada la hipótesis H1 "Las emociones negativas estarán correlacionadas con la intención de compartir una noticia en redes sociales". (Ver gráfico 7).

Respecto a la pregunta de investigación PI2. “¿Qué usos y gratificaciones perseguidos en redes sociales moderarán la influencia de las emociones a la hora de compartir una noticia sobre cambio 
climático?" los datos apuntan a la búsqueda de información como único uso y gratificación que modera el papel de la emoción (ver tabla 6). La gestión de la propia imagen y la interacción social se mantienen al margen de esta influencia.

En cuanto a la PI3 “¿Qué hábitos de consumo informativo en redes sociales moderarán la influencia de las emociones a la hora de compartir una noticia sobre cambio climático?", los resultados de la investigación apuntan a que tan solo el consumo de noticias (internalización de noticias) modera la influencia emocional a la hora de compartir un contenido en redes sociales sobre cambio climático. Por el contrario, el hábito de externalización no influye en este sentido (ver tabla 7).

La respuesta a la PI4. “¿Qué actitudes previas hacia el cambio climático moderarán la influencia de las emociones a la hora de compartir una noticia en redes sociales sobre este asunto?" es negativa en el sentido de que ninguna de estas actitudes modera la influencia de la emoción sobre la decisión de compartir una noticia. De igual manera los datos no permiten verificar la hipótesis H2 "El conocimiento previo sobre el cambio climático moderará la influencia de las emociones sobre la intención de compartir una noticia en redes sociales sobre este asunto". Por el contrario, se cumple la H3 "El grado de preocupación previo sobre el cambio climático moderará la influencia de las emociones sobre la intención de compartir una noticia en redes sociales sobre este asunto" (ver tabla 8).

Los hallazgos presentados van en la línea de otros trabajos que señalaban la importancia del miedo a la hora de despertar la implicación pública en la acción contra el cambio climático (Hartmann, Apaolaza, D'Souza, Barrutia, \& Echebarria, 2014). Este trabajo demuestra que el miedo es también un factor relevante a la hora de que los usuarios den mayor visibilidad a mensajes sobre los efectos y consecuencias del cambio climático. Esta evidencia puede resultar especialmente útil a la hora de diseñar campañas en redes sociales para la concienciación medioambiental en este sentido.

Por otro lado, este estudio también introduce la rabia como factor relevante a la hora de decidir si compartir una noticia sobre cambio climático. Si bien se había señalado en otros contextos como el de la información política (Hasell \& Weeks, 2016) este aspecto emocional todavía no se había señalado en el caso concreto de la comunicación medioambiental y, concretamente, sobre el cambio climático.

En cuanto a los factores considerados como moderadores de este impacto emocional se ha desvelado el papel desempeñado por la búsqueda activa de información en este sentido. Tanto el uso y gratificación de búsqueda de información como el hábito concreto del consumo de noticias mediante redes sociales amortiguan la carga emocional de las noticias. Este hecho es consecuente con el papel de la preocupación por el cambio climático. Es decir, cuanto más preocupado está un usuario en obtener información y más información recibe, menos le afecta la carga emocional de una noticia a la hora de compartirla entre sus contactos. No obstante, el conocimiento previo sobre el cambio climático no modera de igual manera ese impacto emocional. Por lo tanto, puede concluirse que la influencia emocional solo es moderada si existe preocupación o intención de informarse, no si esa información se lleva realmente a cabo y es efectiva en el ámbito concreto del cambio climático.

\subsection{Limitaciones y prospectiva}

El estudio solo ha empleado un mensaje como estímulo para medir la respuesta emocional, con lo que no se han podido comparar los efectos y el papel ejercido por distintos aspectos de la noticia o de su tratamiento.

Se ha señalado que diferentes enfoques y encuadres pueden hacer variar los efectos de los mensajes sobre cambio climático (Bilandzic, Kalch, \& Soentgen, 2017; Feldman \& Hart, 2018) y que estas 
diferencias en el tratamiento de la noticia pueden provocar efectos distintos en las actitudes e intenciones de los usuarios (Hart \& Feldman, 2016). Así, puesto que los usuarios reaccionan de manera diferente ante distintos encuadres (Lee, Chang, \& Chen, 2017) es necesario que estudios posteriores comparen las respuestas de los participantes ante varios estímulos que incluyan distintos enfoques.

Dichos estudios posteriores deberían emplear diferentes estímulos con diferencias sustantivas en estos aspectos para así mediar las variaciones que provocan en la reacción de los lectores.

Por otro lado, los resultados presentados son solo extrapolables a un sector concreto de la población español como son estudiantes universitarios. Futuros trabajos deberían comparar los resultados obtenidos con población de otras edades. En este mismo sentido, puesto que las reacciones ante emociones pueden ser distintas en diferentes entornos culturales (Eriksson, Coultas, \& de Barra, 2016) son necesarias réplicas transculturales y transnacionales de este estudio de modo que se puedan entender mejor los efectos de las emociones percibidas en la difusión secundaria de noticias.

\section{Referencias bibliográficas}

Al-Rawi, A. (2017). Viral News on Social Media. Digital Journalism, 7(1), 1-17. https://doi.org/10.1080/21670811.2017.1387062

Amoedo, A., Vara-Miguel, A. \& Negredo, S. (2018). Digital News Report.es 2018. https://drive.google.com/file/d/1_MqxpbPvMQM1lpvjsGm4QOKxlMC8IZ_D/view

Anderson, A. A. \& Huntington H. E. (2017). Social Media, Science, and Attack Discourse: How Twitter Discussions of Climate Change Use Sarcasm and Incivility. Science Communication, 39(5), 598-620. https://doi.org/10.1177/1075547017735113

Antunovic, D., Parsons, P. \& Cooke, T. R. (2018). 'Checking' and googling: Stages of news consumption among young adults. Journalism, 19(5), 632-648. https://doi.org/10.1177/1464884916663625

Berger, J. \& Milkman K. L. (2012) What Makes Online Content Viral? Journal of Marketing Research, 49(2), 192-205. https://doi.org/10.1509/jmr.10.0353

Bilandzic, H., Kalch, A. \& Soentgen, J. (2017). Effects of Goal Framing and Emotions on Perceived Threat and Willingness to Sacrifice for Climate Change. Science Communication, 39(4), 466-491. https://doi.org/10.1177/1075547017718553

Bobkowski, P. S. (2015). Sharing the News: Effects of Informational Utility and Opinion Leadership on Online News Sharing. Journalism \& Mass Communication Quarterly, 92(2), 320-345. https://doi.org/10.1177/1077699015573194

Boulianne, S (2015). Social media use and participation: A meta-analysis of current research. Information, Communication \& Society, 18(5), 524-538.

Bright, J. (2016). The Social News Gap: How News Reading and News Sharing Diverge. Journal of Communication, 66(3), 343-365. https://doi.org/10.1111/jcom.12232 
Cappella, J. N., Kim, H. S. \& Albarracín, D. (2015). Selection and Transmission Processes for Information in the Emerging Media Environment: Psychological Motives and Message Characteristics. Media Psychology, 18(3), 396-424.

https://doi.org/10.1080/15213269.2014.941112

Carvalho, A., Van Wessel, A. \& Maeseele, P. (2017). Communication practices and political engagement with climate change: A research agenda. Environmental Communication, 11(1), 122-135.

Chadwick, A. E. (2015). Toward a Theory of Persuasive Hope: Effects of Cognitive Appraisals, Hope Appeals, and Hope in the Context of Climate Change. Health Communication, 30(6), 598611. https://doi.org/10.1080/10410236.2014.916777

Chen, M. F. (2016). Impact of fear appeals on pro-environmental behavior and crucial determinants. International Journal of Advertising, 35(1), 74-92.

https://doi.org/10.1080/02650487.2015.1101908

Choi, J. (2016a). News Internalizing and Externalizing: The Dimensions of News Sharing on Online Social Networking Sites. Journalism \& Mass Communication Quarterly, 93(4), 816-835. https://doi.org/10.1177/1077699016628812

Choi, J. (2016b). Why do people use news differently on SNSs? An investigation of the role of motivations, media repertoires, and technology cluster on citizens' news-related activities. Computers in Human Behavior, 54, 249-256. https://doi.org/10.1016/j.chb.2015.08.006

Choi, J. \& Lee, J. K. (2015). Investigating the effects of news sharing and political interest on social media network heterogeneity. Computers in Human Behavior, 44, 258-266. https://doi.org/10.1016/j.chb.2014.11.029

Chyi, H. I. \& Yang, M. J. (2009). Is online news an inferior good? Examining the economic nature of online news among users. Journalism \& Mass Communication Quarterly, 86(3), 594-612. https://doi.org/10.1177/107769900908600309

Coppini, D., Duncan, M. A., Mcleod, D. M., Wise, D. A., Bialik, K. E. \& Wu, Y. (2017). When the whole world is watching: A motivations-based account of selective expression and exposure. Computers in Human Behavior, 75, 766-774. https://doi.org/10.1016/j.chb.2017.04.020

Da Silva, J. P. \& Pereira, A. M. S. (2017). Perceived Spirituality, Mindfulness and Quality of Life in Psychiatric Patients. Journal of Religion and Health, 56(1), 130-140. https://doi.org/10.1007/s10943-016-0186-y

Dafonte-Gómez, A. (2018). News Media and the Emotional Public Sphere| Audiences as Medium: Motivations and Emotions in News Sharing. International Journal of Communication, 12, 2.1332.152. https://doi.org/1932-8036/20180005

Eriksson, K., Coultas, J. C. \& De Barra, M. (2016). Cross-Cultural Differences in Emotional Selection on Transmission of Information. Journal of Cognition and Culture, 16(1-2), 122-143. https://doi.org/10.1163/15685373-12342171

Feldman, L. \& Hart, P. S. (2016). Using Political Efficacy Messages to Increase Climate Activism. Science Communication, 38(1), 99-127. https://doi.org/10.1177/1075547015617941 
Feldman, L. \& Hart, P. S. (2018). Is There Any Hope? How Climate Change News Imagery and Text Influence Audience Emotions and Support for Climate Mitigation Policies. Risk Analysis, 38(3), 585-602. https://doi.org/10.1111/risa.12868

Gao, Q. \& Feng, C. (2016). Branding with social media: User gratifications, usage patterns, and brand message content strategies. Computers in Human Behavior, 63, 868-890. https://doi.org/10.1016/j.chb.2016.06.022

Gerber, A. H., McCormick, C. E. B., Levine, T. P., Morrow, E. M., Anders, T. F. \& Sheinkopf, S. J. (2017). Brief Report: Factors Influencing Healthcare Satisfaction in Adults with Autism Spectrum Disorder. Journal of Autism and Developmental Disorders, 47(6), 1.896-1.903. https://doi.org/10.1007/s10803-017-3087-3

Gil de Zúñiga, H., Jung, N. \& Valenzuela, S. (2012). Social Media Use for News and Individuals' Social Capital, Civic Engagement and Political Participation. Journal of Computer-Mediated Communication, 17(3), 319-336. https://doi.org/10.1111/j.1083-6101.2012.01574.x

Gil de Zúñiga, G., Molyneux, L. \& Zheng, P. (2014). Social media, political expression, and political participation: Panel analysis of lagged and concurrent relationships. Journal of Communication, 64(4), 612-634.

Guallar, J., Suau, J., Ruiz-Caballero, C., Sáez, A. \& Masip, P. (2016) Re-dissemination of news and public debate on social networks. El profesional de la información, 25(3), 1.699-2.407. https://doi.org/10.3145/epi.2016.may.05

Harber, K. D. \& Cohen, D. J. (2005). The Emotional Broadcaster Theory of Social Sharing. Journal of Language and Social Psychology, 24(4), 382-400. https://doi.org/10.1177/0261927X05281426

Hart, P. S. \& Feldman, L. (2016). The Influence of Climate Change Efficacy Messages and Efficacy Beliefs on Intended Political Participation. PLOS ONE, 11(8), e0157658. https://doi.org/10.1371/journal.pone.0157658

Hartmann, P., Apaolaza, V., D’Souza, C., Barrutia, J. M. \& Echebarria, C. (2014). Environmental threat appeals in green advertising. International Journal of Advertising, 33(4), 741-765. https://doi.org/10.2501/IJA-33-4-741-765

Hasell, A. \& Weeks, B. E. (2016) Partisan Provocation: The Role of Partisan News Use and Emotional Responses in Political Information Sharing in Social Media! Human Communication Research, 42(4), 641-661. https://doi.org/10.1111/hcre.12092

Höijer, B. (2010). Emotional anchoring and objectification in the media reporting on climate change, Public Understanding of Science, 19(6), 717-731. https://doi.org/10.1177/0963662509348863

Hopke, J. E. \& Hestres, L. E. (2018). Visualizing the Paris Climate Talks on Twitter: Media and Climate Stakeholder Visual Social Media During COP21. Social Media + Society, 4(3), 205630511878268. https://doi.org/10.1177/2056305118782687

Hossain, M. A., Dwivedi, Y. K., Chan, C., Standing, C. \& Olanrewaju, A. S. (2018). Sharing political content in online social media: A planned and unplanned behaviour approach. Information Systems Frontiers, 20(3), 485-501. 
Hyun, K. D. \& Kim, J. (2015). Differential and interactive influences on political participation by different types of news activities and political conversation through social media. Computers in Human Behavior, 45, 328-334. https://doi.org/10.1016/j.chb.2014.12.031

Kalsnes, B. \& Larsson, A. O. (2018). Understanding News Sharing Across Social Media. Journalism Studies, 19(11), 1.669-1.688. https://doi.org/10.1080/1461670X.2017.1297686

Karnowski, V., Kümpel, A. S., Leonhard, L. \& Leiner, D. J. (2017). From incidental news exposure to news engagement. How perceptions of the news post and news usage patterns influence engagement with news articles encountered on Facebook. Computer Human Behaviour, 76, 4250. https://doi.org/10.1016/j.chb.2017.06.041

Karnowski, V., Leonhard, L. \& Kümpel A. S. (2018). Why Users Share the News: A Theory of Reasoned Action-Based Study on the Antecedents of News-Sharing Behavior. Communication Research Reports, 35(2), 91-100.

Katz-Kimchi, M. \& Manosevitch, I. (2015). Mobilizing Facebook Users against Facebook's Energy Policy: The Case of Greenpeace Unfriend Coal Campaign. Environmental Communication, 9(2), 248-267. https://doi.org/10.1080/17524032.2014.993413

Kauffmann, Y., Ramel, J. C., Lefebvre, A., Isaico, R., De Lazzer, A., Bonnabel, A., Bron, A. M. \& Creuzot-Garcher, C. (2015). Preoperative Prognostic Factors and Predictive Score in Patients Operated on for Combined Cataract and Idiopathic Epiretinal Membrane. American Journal of Ophthalmology, 160(1), 185-192. https://doi.org/10.1016/J.AJO.2015.03.027

Keib, K., Himelboim, I. \& Han, J. Y. (2018). Important tweets matter: Predicting retweets in the \#BlackLivesMatter talk on twitter. Computers in Human Behavior, 85, 106-115. https://doi.org/10.1016/j.chb.2018.03.025

Kilgo, D. K., Lough, K. \& Riedl, M. J. (2017). Emotional appeals and news values as factors of shareworthiness in Ice Bucket Challenge coverage. Digital Journalism, 1-20. https://doi.org/10.1080/21670811.2017.1387501

Kirk, A. H. P., Ng, B. S. P., Lee, A. N., Ang, B. \& Lee, J. H. (2015). Perceptions of Pediatric Critical Care Nurses on the Initiation of a Nursing-Led Feeding Protocol. Journal of Nursing Research, 23(4), 308-12. https://doi.org/10.1097/jnr.0000000000000085

Klinger, U. \& Svensson, J. (2015). The emergence of network media logic in political communication: A theoretical approach. New media \& society, 17(8), 1.241-1.257. https://doi.org/10.1177/1461444814522952

Korgaonkar, P. K. \& Wolin, L. D. (1999). A multivariate analysis of web usage. Journal of Advertising Research, 39(2), 53-68.

Krämer, N. C. \& Winter, S. (2008). Impression Management 2.0. The Relationship of Self-Esteem, Extraversion, Self-Efficacy, and Self-Presentation Within Social Networking Sites. Journal of Media Psychology, 20(3), 106-116. https://doi.org/10.1027/1864-1105.20.3.106

LaRose, R. \& Eastin, M. S. (2004). A social cognitive theory of Internet uses and gratifications: Toward a new model of media attendance. Journal of Broadcasting \& Electronic Media, 48(3), 358-377. 
Larsson, A. O. (2018). "I Shared the News Today, oh Boy". News provision and interaction on Facebook. Journalism Studies, 19(1), 43-61. https://doi.org/10.1080/1461670X.2016.1154797

Lee, C. S. \& Ma, L. (2012). News sharing in social media: The effect of gratifications and prior experience. Computers in Human Behavior, 28(2), 331-339.

https://doi.org/10.1016/j.chb.2011.10.002

Lee, N. M., VanDyke, M. S. \& Cummins, R. G. (2018). A Missed Opportunity?: NOAA's Use of Social Media to Communicate Climate Science. Environmental Communication, 12(2), 274-283. https://doi.org/10.1080/17524032.2016.1269825

Lee, Y. K., Chang, C. T. \& Chen, P. C. (2017). What Sells Better in Green Communications: Fear or Hope?. Journal of Advertising Research, 57(4), 379-396. https://doi.org/10.2501/JAR-2017-048

Lischka, J. A. (2018). Logics in social media news making: How social media editors marry the Facebook logic with journalistic standards. Journalism, 146488491878847. https://doi.org/10.1177/1464884918788472

Martín-Quevedo, J., Fernández-Gómez, E. \& Segado-Boj, F. (2019). How to Engage with Younger Users on Instagram: A Comparative Analysis of HBO and Netflix in the Spanish and US Markets. International Journal of Media Management, 1-21. https://doi.org/10.1080/14241277.2019.1585355

Masip, P., Guallar, J., Suau, J., Ruiz-Caballero, C. \& Peralta, M. (2015). News and social networks: audience behavior. El profesional de la información, 4(4), 363-370.

McLeod, J. M. (2000). Media and civic socialization of youth. Journal of Adolescent Health, 27(2), 45-51. https://doi.org/10.1016/S1054-139X(00)00131-2

Nabi, R. L., Gustafson, A. \& Jensen, R. (2018). Framing Climate Change: Exploring the Role of Emotion in Generating Advocacy Behavior. Science Communication, 40(4), 442-468. https://doi.org/10.1177/1075547018776019

Oakley, R. L. \& Salam, A. F. (2014). Examining the impact of computer-mediated social networks on individual consumerism environmental behaviors. Computers in human behavior, 35, 516-526.

Östman, J. (2014). The influence of media use on environmental engagement: A political socialization approach. Environmental Communication, 8(1), 92-109.

Penney, E. S. \& Abbott, M. J. (2015). The Impact of Perceived Standards on State Anxiety, Appraisal Processes, and Negative Pre- and Post-event Rumination in Social Anxiety Disorder. Cognitive Therapy and Research, 39(2), 162-177. https://doi.org/10.1007/s10608-014-9639-3

Scherman, A., Arriagada, A. \& Valenzuela, S. (2015). Student and environmental protests in Chile: The role of social media. Politics, 35(2), 151-171.

Shapiro M. A. \& Park, W. H. (2018). Climate Change and YouTube: Deliberation Potential in Post-video Discussions. Environmental Communication, 12(1), 115-131. https://doi.org/10.1080/17524032.2017.1289108 
Singer, J. B. (2014). User-generated visibility: Secondary gatekeeping in a shared media space. New Media \& Society, 16(1), 55-73. https://doi.org/10.1177/1461444813477833

Skoric, M. M., Zhu, Q., Goh, D. \& Pang, N. (2016). Social media and citizen engagement: A metaanalytic review. New Media \& Society, 18(9), 1.817-1.839.

Stempel, C., Hargrove, T. \& Stempel, G. H. (2007). Media Use, Social Structure, and Belief in 9/11 Conspiracy Theories. Journalism \& Mass Communication Quarterly, 84(2), 353-372. https://doi.org/10.1177/107769900708400210

Stieglitz, S. \& Dang-Xuan, L. (2013). Emotions and Information Diffusion in Social MediaSentiment of Microblogs and Sharing Behavior. Journal of Management Information Systems, 29(4), 217-248. https://doi.org/10.2753/MIS0742-1222290408

Taddicken, M. \& Reif, A. (2016). Who participates in the climate change online discourse? A typology of Germans' online engagement. Communications, 41(3), 315-337. https://doi.org/10.1515/commun-2016-0012

Thorson, K., Edgerly, S., Kligler-Vilenchik, N., Xu, Y. \& Wang, L. (2016). Seeking visibility in a big tent: Digital communication and the people's climate march. International Journal of Communication, 10, 4.784-4.806. http://ijoc.org/index.php/ijoc/article/view/4703/1802

Van der Wurff, R. \& Schoenbach, K. (2014). Civic and citizen demands of news media and journalists: What does the audience expect from good journalism? Journalism \& Mass Communication Quarterly, 91(3), 433-451.

La Vanguardia (2017). Este río ha desaparecido y los científicos culpan al cambio climático. 19-42017. https://www.lavanguardia.com/natural/20170418/421794268373/rio-desaparece-cambioclimatico.html.

Velásquez, A., Renó, D., Beltrán, A. M., Maldonado, J. C. y Ortiz León, C. (2018). De los mass media a los medios sociales: reflexiones sobre la nueva ecología de los medios. Revista Latina de Comunicación Social, 73, 583-594.

Villi, M. \& Matikainen, J. (2015). Mobile UDC: Online media content distribution among Finnish mobile Internet users. Mobile Media \& Communication, 3(2), 214-229. https://doi.org/10.1177/2050157914552156

Villi, M. \& Noguera-Vivo, J. M. (2017). Sharing media content in social media: The challenges and opportunities of user-distributed content (UDC). Journal of Applied Journalism \& Media Studies, 6(2), 207-223. https://doi.org/10.1386/ajms.6.2.207_1

Weeks, B. E. \& Holbert, R. L. (2013). Predicting Dissemination of News Content in Social Media: A Focus on Reception, Friending, and Partisanship. Journalism \& Mass Communication Quarterly, 90(2), 212-232. https://doi.org/10.1177/1077699013482906

Whiting, A. \& Williams, D. (2013). Why people use social media: a uses and gratifications approach. Qualitative Market Research: An International Journal, 16(4), 362-369. https://doi.org/10.1108/QMR-06-2013-0041 
Winter, S., Metzger, M. J. \& Flanagin, A. J. (2016). Selective Use of News Cues: A Multiple-Motive Perspective on Information Selection in Social Media Environments. Journal of Communication, 66(4), 669-693. https://doi.org/10.1111/jcom.12241

Wirz, D. S. (2018). Persuasion Through Emotion? An Experimental Test of the Emotion-Eliciting Nature of Populist Communication. International Journal of Communication, 12, 1.114-1.138. http://ijoc.org/index.php/ijoc/article/view/7846

Yang, Z. J., Kahlor, L. A. \& Griffin, D. J. (2014). I Share, Therefore I Am: A U.S.-China Comparison of College Students' Motivations to Share Information About Climate Change. Human Communication Research, 40(1), 112-135. https://doi.org/10.1111/hcre.12018

Zhang, N. \& Skoric, M. M. (2018). Media Use and Environmental Engagement: Examining Differential Gains from News Media and Social Media. International Journal of Communication, 12, 380-403. http://ijoc.org/index.php/ijoc/article/view/7650

\section{AUTORES:}

\section{Francisco Segado-Boj}

Profesor Titular de Universidad acreditado. Escuela de Doctorado. Universidad Internacional de La Rioja. España.

Doctor en Periodismo por la Universidad Complutense de Madrid (2008). En la actualidad es profesor en la Universidad Internacional de la Rioja (UNIR), donde dirige el Grupo de Investigación "Comunicación y Sociedad Digital". Acreditado para la figura de Profesor Titular de Universidad, cuenta con dos sexenios de investigación reconocidos por la CNEAI. Sus líneas de investigación se centran en los medios digitales y en las redes sociales, así como en la comunicación científica y académica. Ha publicado varias decenas de artículos sobre estos temas en revistas como Telematics \& Informatics, Comunicar, El Profesional de la Información, Journal of Scholarly Publishing o First Monday. En la actualidad es investigador principal del Proyecto "Consumo de noticias en medios sociales. Análisis de factores en la selección y difusión de contenidos mediáticos", referencia CSO2017-86312-R financiado por el Ministerio de Economía, Industria y Competitividad (MINECO), la Agencia Estatal de Investigación (AEI) y el Fondo Europeo de Desarrollo Regional (FEDER).

francisco.segado@unir.net

Índice $\mathbf{H}: 9$

Orcid ID: http://orcid.org/0000-0001-7750-3755

Google Scholar: https://scholar.google.es/citations?user=pY9m6isAAAAJ\&hl=es

\section{Jesús Díaz-Campo}

Profesor Titular de Universidad acreditado. Departamento de Comunicación. Facultad de Empresa y Comunicación. Universidad Internacional de La Rioja. España.

Licenciado en Periodismo y Doctor en Comunicación por la Universidad Complutense de Madrid. Obtuvo una Beca de Formación del Profesorado del Ministerio de Educación y Cultura y realizó estancias de investigación en el Journalism and Mass Communication Department of Tampere University (Finlandia) y en las sedes de la European Journalism Training Association (Tilburg, Holanda) y del European Journalism Centre (Maastricht, Holanda). Es Profesor en la Facultad de Empresa y Comunicación de la Universidad Internacional de La Rioja (UNIR), donde dirige el Máster Universitario en Comunicación e Identidad Corporativa e imparte varias asignaturas desde 2011. También ha impartido docencia en la Universidad Complutense de Madrid (como becario 
FPU) y en la Universidad Pontificia Comillas. Está acreditado por ANECA a profesor titular de universidad. Miembro del Grupo de Investigación Comunicación y Sociedad Digital (COYSODI) de la Universidad Internacional de la Rioja. Es autor de más de cuarenta y artículos publicados en diversas revistas académicas (Telematics \&Informatics, Transinformaçao, El Profesional de la Información, Revista Latina de Comunicación Social, Estudios sobre el Mensaje Periodístico, Palabra Clave, Cuadernos.Info, Observatorio (OBS) o Historia y Comunicación Social, entre otras). Además, ha participado en varios proyectos de investigación obtenidos en régimen competitivo y tiene un sexenio de investigación reconocido por la CNEAI. Sus principales líneas de investigación se centran en Ética de la Comunicación; Responsabilidad Social Corporativa; Comunicación Política y Redes Sociales; Radio.

jesus.diaz@unir.net

Índice H: 11

Orcid ID: https://orcid.org/0000-0001-5014-8749

Google Scholar: https://scholar.google.es/citations? user=rNxx5WYAAAAJ\&hl=es

\section{Nuria Navarro-Sierra}

Profesora asociada. Departamento de Ciencias de la Comunicación y Sociología. Facultad Ciencias de la Comunicación. Universidad Rey Juan Carlos. España.

Licenciada y Doctora con mención internacional en Comunicación Audiovisual por la Universidad Complutense de Madrid. Estuvo contratada como becaria en el Dpto. de Comunicación y Publicidad I a través del programa de Formación del Profesorado Universitario del Ministerio de Educación, Cultura y Deporte y realizó una estancia de investigación en la University of Lincoln (UK). Es profesora Asociada en los grados y dobles grados de Periodismo, Comunicación Audiovisual y Periodismo. Anteriormente ha impartido docencia en la Universidad Complutense de Madrid y el Centro de Estudios Superiores Felipe II, en los grados y licenciaturas de Comunicación Audiovisual. Es miembro del Grupo de Investigación Visual, vinculado a la Universidad Rey Juan Carlos (España). Entre sus publicaciones se encuentran líneas de investigación referentes a la televisión y radio en España, nuevos modelos de negocio o narrativa de los nuevos medios de comunicación digital.

nuria.navarro.sierra@urjc.es

Índice $\mathrm{H}: 3$

Orcid ID: https://orcid.org/0000-0002-1431-1534

Google Scholar: https://scholar.google.es/citations?user=qP7Y1 oAAAAJ\&hl=es 